\title{
Visualization of regional cerebrospinal fluid flow with a dye injection technique in focal arachnoid pathologies
}

\author{
Satoshi Yamaguchi, MD, PhD, ${ }^{1}$ Kazutoshi Hida, MD, PhD, ${ }^{2}$ Masaaki Takeda, MD, PhD, ${ }^{1}$ \\ Takafumi Mitsuhara, MD, PhD, ${ }^{1}$ Mizuki Morishige, ${ }^{3}$ Naoto Yamada, MD, ${ }^{1}$ and \\ Kaoru Kurisu, MD, PhD'
}

\begin{abstract}
'Department of Neurosurgery, Hiroshima University Graduate School of Biomedical and Health Sciences, Hiroshima; ${ }^{3}$ Division of Clinical Engineering, Hiroshima University Hospital, Hiroshima; and ²Department of Neurosurgery, Sapporo Azabu Neurosurgical Hospital, Sapporo, Hokkaido, Japan
\end{abstract}

\begin{abstract}
Surgical lysis of the thickened arachnoid membrane is the first choice of treatment in spinal arachnoid pathologies that cause flow disturbances or blockage of CSF. However, it is important to consider that while extensive lysis of the arachnoid may temporarily provide a wide pathway for CSF, an extensive lytic procedure may later cause secondary adhesion. Thus, it is ideal for the proper extent of the arachnoid lysis to be determined after careful analysis of regional CSF flow. The authors report their limited experience with intraoperative visualization of CSF flow in spinal arachnoid pathologies. Two patients with a dorsal arachnoid web (DAW) with cervical syringomyelia and 1 patient with focal adhesive arachnoiditis causing edema of the spinal cord were surgically treated at the authors' institution between 2007 and 2013. In all cases, the presence of a DAW or focal adhesive arachnoiditis was suspected from the findings on MRI, namely 1) an indentation on the upper thoracic spinal cord and 2) syringomyelia and/or edema of the spinal cord above the indentation. Exploratory surgery disclosed a transversely thickened arachnoid septum on the dorsal side of the indented cord. To prove blockage of the CSF by the septum and to decide on the extent of arachnoid lysis, regional CSF flow around the arachnoid septum was visualized by subarachnoid injection of gentian violet solution close to the web. Injected dye stagnated just close to the arachnoid septum in all cases, and these findings documented CSF blockage by the septum. In 2 cases, a 2-minute observation showed that the injected dye stayed close to the web without diffusion. The authors performed not only resection of the web itself but also lysis of the thickened arachnoid on both sides of the spinal cord to make a CSF pathway on the ventral side. In the third case, the dye stagnated close to the web at first but then diffused through the nerve root to the ventral CSF space. The lysis procedure was completed after exclusive removal of the dorsal web. Postoperative MR images confirmed reduction of the syrinx and/or improvement of the edema in all cases, suggesting that the extent of arachnoid lysis was optimal in each case. No adverse effect was observed after subarachnoid injection of gentian violet. The authors believe that their technique of visualizing regional CSF flow will be helpful to decide the optimal extent of lysis in some local arachnoid pathologies.
\end{abstract}

http://thejns.org/doi/abs/10.3171/2014.10.SPINE1446

KEY WORDS dorsal arachnoid web; focal adhesive arachnoiditis; lysis; gentian violet; technique

$\mathrm{S}$ URGICAL lysis of a thickened arachnoid is the first choice of treatment for spinal arachnoid pathologies that cause flow disturbance or blockage of CSF.,11 While extensive arachnoid lysis may temporarily provide a wide pathway for CSF, such extensive lysis may later cause secondary adhesion. ${ }^{7}$ Thus, it is ideal for the proper amount of microlysis of the thickened arachnoid membrane to be determined after assessment of regional CSF flow. Advanced imaging modalities such as multislice
CT-based myelography and MRI-based CSF flow analysis may demonstrate flow disturbance or blockage of CSF along the spinal cord ${ }^{6,9}$ However, a method of intraoperative evaluation of regional CSF flow around the arachnoid lesion has not been described before. In the present article, we introduce a simple technique to visualize regional CSF flow with the aid of a gentian violet solution. We report our use of and limited experience with this dye injection technique. 


\section{Surgical Technique}

Between October 2007 and September 2013, 2 cases of a surgically confirmed dorsal arachnoid web (DAW) and 1 case of focal arachnoiditis presented with myelopathy. The age of the patients at onset ranged from 38 to 56 years old. The etiology of arachnoid septum was idiopathic in the cases of DAW and subarachnoid hemorrhage in the case of focal arachnoiditis (Table 1). In all cases, preoperative MRI showed an indentation in the upper thoracic spinal cord, and syrinx formation and/or edema of the spinal cord was confirmed above the indentation (Fig. 1A, C, and E). Although multiplanar reformatted sagittal CT myelography also delineated the contour of the compressed spinal cord,
TABLE 1. Summary of the present cases

\begin{tabular}{ccclc}
\hline $\begin{array}{c}\text { Case } \\
\text { No. }\end{array}$ & $\begin{array}{c}\text { Age (yrs), } \\
\text { Sex }\end{array}$ & Arachnoid Pathology & Etiology & Septum Level \\
\hline 1 & $56, \mathrm{M}$ & DAW & Idiopathic & T3-4 \\
\hline 2 & $38, \mathrm{M}$ & Arachnoiditis & SAH & T3-4 \\
\hline 3 & $52, \mathrm{M}$ & DAW & Idiopathic & T-1 \\
\hline
\end{tabular}

$\mathrm{SAH}=$ subarachnoid hemorrhage.

it could not demonstrate the relevant lesion causing compression on the cord. We suspected that some focal arachnoid pathology, such as focal arachnoiditis or an arachnoid cyst, had hindered CSF flow behind the cord and caused
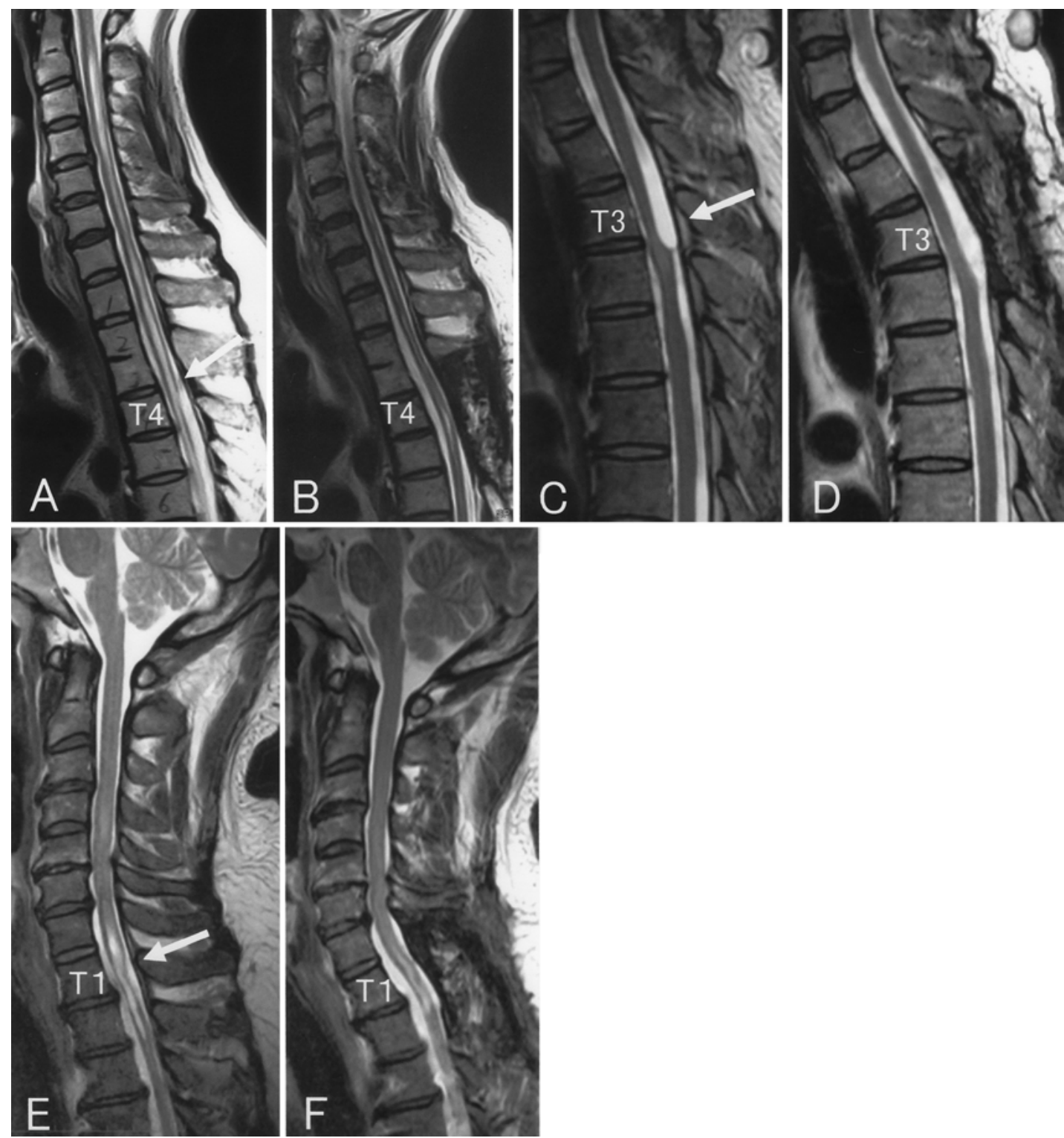

FIG. 1. Preoperative $(A, C$, and $E$ ) and postoperative $(B, D$, and $F) M R$ images obtained in the 3 patients. $A$ and $B$ : Case 1. Preoperative MR image (A) showing indentation (arrow) at the T3-4 level and syringomyelia in the cervicothoracic cord. Postoperative MR image (B) obtained 11 months after the operation, showing shrinkage of the syrinx. C and D: Case 2. Preoperative MR image (C) showing deformity of the spinal cord (arrow) at the T3-4 level and edema of the indented cord. Postoperative MR image (D) obtained 1 year after the operation, confirming improvement of deformity and edema of the thoracic cord. E and F: Case 3. Preoperative MR image (E) showing indentation at T-1 (arrow). Syringomyelia is seen above and below the indentation. Postoperative MR image (F) obtained 10 months after the operation, showing improvement of indentation and syrinx. 
secondary syringomyelia. Surgical exploration in the site of the indentation disclosed the presence of a DAW (Cases 1 and 3) and the presence of the septum caused by focal adhesive arachnoiditis (Case 2). After evaluating regional CSF flow by the dye injection technique, microlysis of the thickened arachnoid membrane was performed.

Before the lysis procedure, the dye injection technique was performed to evaluate regional CSF flow around the focally thickened arachnoid. After a several-level laminectomy, the dura was opened with special care not to cut the arachnoid membrane. At the indentation, the arachnoid was focally thickened in the transverse direction. We prepared the dye by diluting gentian violet 500 times with physiological saline: $0.02 \mathrm{ml}$ of gentian violet in $10 \mathrm{ml}$ of physiological saline (Fig. 2A). To evaluate regional CSF flow, we injected $0.1-0.2 \mathrm{ml}$ of the gentian violet solution with a 26-gauge needle into the subarachnoid space just caudal to the web. In Cases 1 and 2, flow of the injected dye was blocked by the thickened arachnoid. This finding documented the presence of arachnoid septum and CSF blockage by the septum behind the spinal cord. The amount of injected dye did not decrease during our 2-minute observation. These findings suggested that blockage of the CSF existed not only dorsal to but also lateral to the cord. Thus, we removed the dorsal arachnoid septum and lysed the arachnoid on the lateral sides of the spinal cord.
In Case 3, stagnation of the injected dye just caudal to the thickened arachnoid documented the presence of DAW. However, the dye then diminished gradually as it moved through the nerve roots to the ventral subarachnoid space (Fig. 2B-E). These findings suggested that CSF flow had a route on either side of the cord. The lysis procedure was completed after exclusive removal of the dorsal web.

\section{Results}

In Case 1, postoperative MR images obtained 11 months after surgery showed reduction of the syrinx (Fig. 1B). The patient's numbness did not change, and the patient was lost to follow-up. In Case 2, numbness in the lower limbs and a constriction sensation around the trunk disappeared after surgery. MR images obtained 1 year after the operation showed improvement of deformity and edema of the spinal cord (Fig. 1D). In Case 3, the patient's numbness improved. MR images obtained 10 months after the operation showed the syrinx had markedly reduced in size (Fig. 1F).

\section{Discussion}

Arachnoid cysts and arachnoiditis are typical spinal arachnoid pathologies encountered in neurosurgical practice. When such arachnoid lesions block the pathway of the CSF, syringes and/or edema may arise in the spinal cord
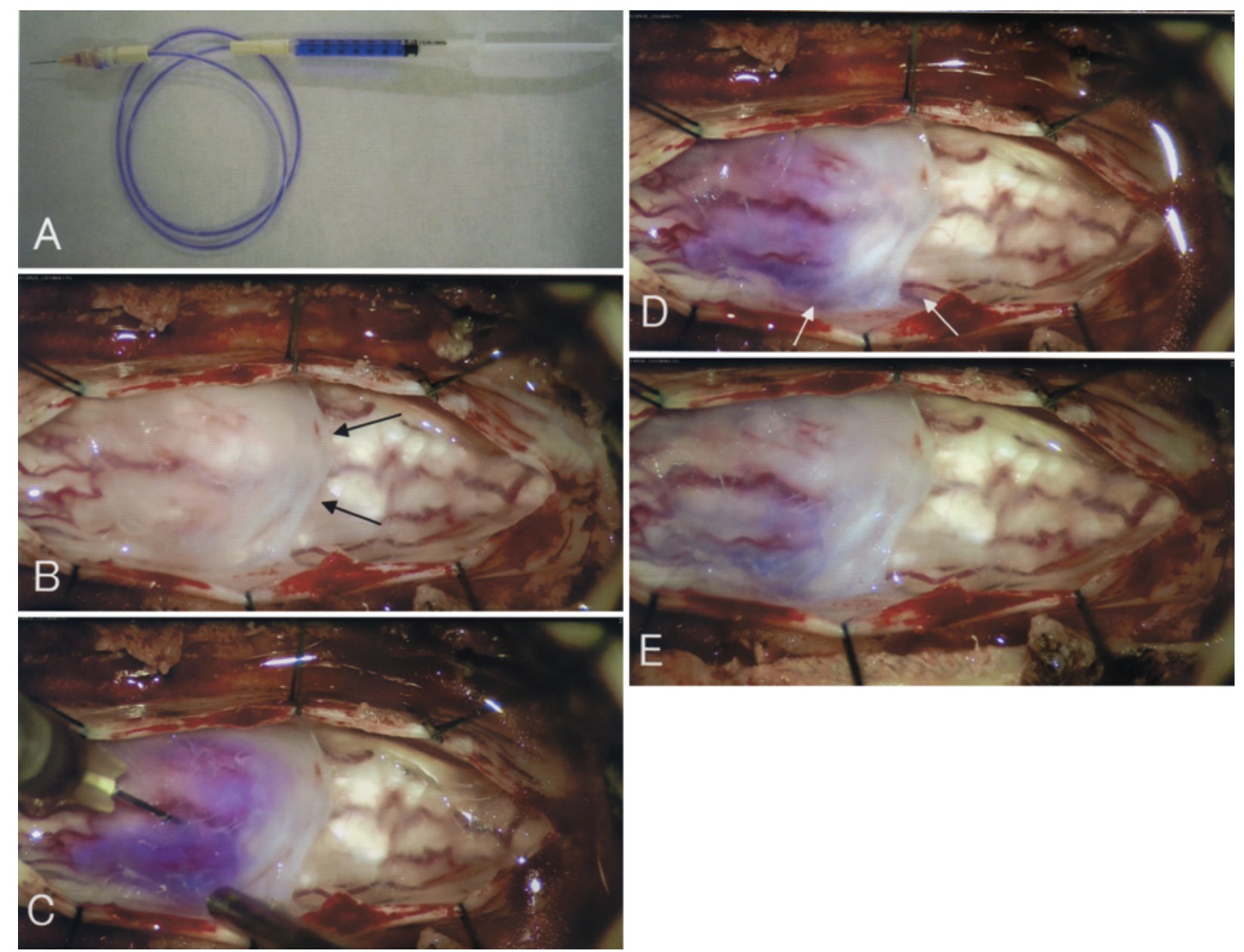

FIG. 2. Preparation of gentian violet solution and serial intraoperative photographs of the dye injection technique. A: Prepared $1.0 \mathrm{ml}$ of gentian violet solution in a syringe and 26-gauge needle. B: Intraoperative photograph obtained after the dural incision, showing transversely thickened arachnoid suggesting a DAW (arrows). C-E: Serial photographs showing diffusion of injected dye into the ventral subarachnoid space. Note that injected dye is diffusing into the ventral subarachnoid space through nerve roots (arrows). 
above or below the lesions. Recent articles have reported DAWs as another arachnoid pathology causing blockage of the CSF and secondary syringomyelia. When we add the present 2 cases (Cases 1 and 3), only 13 cases of surgically confirmed DAWs have been reported..$^{1,8-10}$ Regional CSF circulation of DAWs has rarely been mentioned and has not been fully understood in the past.

Even advanced imaging modalities such as multislice $\mathrm{CT}$ and MRI have difficulty visualizing arachnoid lesions except for large arachnoid cysts. ${ }^{2,9}$ Thus, the presence of focal arachnoid pathologies may be only suspected from imaging findings such as deformity of the spinal cord and blockage of CSF flow. After the relevant lesion causing CSF blockage is located on MR images or CT myelograms, surgical lysis of the pathological arachnoid is indicated to release the blockage of the CSF pathway. However, the lysis procedure introduces the possibility that extensive lysis may temporarily provide a wide pathway for CSF flow but may later lead to adhesion of the arachnoid and relapse of CSF flow disturbance. Assessment of regional CSF flow may suggest the optimal amount of arachnoid lysis. To the best of our knowledge, we are the first to report a simple method of intraoperative assessment of regional CSF flow around DAWs. Stagnation of the dye close to the focal arachnoid lesion clearly proved the presence of the septum and CSF blockage in all cases. In Cases 1 and 2, injected dye showed stagnation close to the web and demonstrated no diffusional CSF flow into the ventral side of the spinal cord. Thus, we performed not only resection of the web itself but also lysis of the thickened arachnoid on both sides of the spinal cord to create a CSF pathway to the ventral side. In Case 3, regional CSF flow visualized after the dye injection demonstrated the web blocking the CSF flow on the dorsal side of the spinal cord, but the dye diminished gradually by moving thorough the nerve roots to the ventral side. The lysis procedure was completed after exclusive removal of the dorsal web. Shrinkage of the syrinx and improvement of spinal cord edema demonstrated on MR images after the operation confirmed that the extent of the lysis was optimal in each case. We used gentian violet for visualization of CSF because it is safe, easily available, and widely used with a long history of topical use in surgical fields as a marker and sometimes as an antiseptic. ${ }^{5}$ With regard to neurosurgical practices, gentian violet has been used for visualization of ostium of the arteriotomy in vascular anastomosis. ${ }^{4}$ In our technique, the trace of the gentian violet is subtle because the dye is diluted 500 times by physiological saline. Although our experience is very limited, so far no adverse effect caused by gentian violet has been encountered.

The limitations of this study are the small number of cases and lack of long-term follow-up. The dye injection technique itself cannot eliminate the possibility of recurrence because any lytic procedure on any focally thickened arachnoid pathology may cause scarring and recurrence. Although further validation after longer follow-up is required to assess the relevance of this technique, we believe that our technique for visualizing regional CSF flow deserves reporting, and the technique will be helpful in deciding the optimal extent of lysis in some local arachnoid pathology.

\section{Conclusions}

We introduced a simple technique using a gentian violet solution that enables intraoperative evaluation of regional CSF flow around arachnoid pathology. Since extensive lysis of a thickened arachnoid may lead to adhesion and relapse of CSF blockage, the optimal and proper range of the lysis should be carefully considered based on intraoperative assessment of regional CSF flow.

\section{References}

1. Brodbelt AR, Stoodley MA: Syringomyelia and the arachnoid web. Acta Neurochir (Wien) 145:707-711, 2003

2. Griessenauer CJ, Bauer DF, Moore TA II, Pritchard PR, Hadley MN: Surgical manifestations of thoracic arachnoid pathology: series of 28 cases. J Neurosurg Spine 20:30-40, 2014

3. Ishizaka S, Hayashi K, Otsuka M, Fukuda S, Tsunoda K, Ushijima R, et al: Syringomyelia and arachnoid cysts associated with spinal arachnoiditis following subarachnoid hemorrhage. Neurol Med Chir (Tokyo) 52:686-690, 2012

4. Kamiyama H, Takahashi A, Houkin K, Mabuchi S, Abe H: Visualization of the ostium of an arteriotomy in bypass surgery. Neurosurgery 33:1109-1110, 1993

5. Maley AM, Arbiser JL: Gentian violet: a 19th century drug re-emerges in the 21st century. Exp Dermatol 22:775-780, 2013

6. Mauer UM, Freude G, Danz B, Kunz U: Cardiac-gated phase-contrast magnetic resonance imaging of cerebrospinal fluid flow in the diagnosis of idiopathic syringomyelia. Neurosurgery 63:1139-1144, 2008

7. Ohata K, Gotoh T, Matsusaka Y, Morino M, Tsuyuguchi N, Sheikh B, et al: Surgical management of syringomyelia associated with spinal adhesive arachnoiditis. J Clin Neurosci 8:40-42, 2001

8. Paramore CG: Dorsal arachnoid web with spinal cord compression: variant of an arachnoid cyst? Report of two cases. J Neurosurg (2 Suppl) 93:287-290, 2000

9. Reardon MA, Raghavan P, Carpenter-Bailey K, Mukherjee S, Smith JS, Matsumoto JA, et al: Dorsal thoracic arachnoid web and the "scalpel sign": a distinct clinical-radiologic entity. AJNR Am J Neuroradiol 34:1104-1110, 2013

10. Sridharan A, Heilman CB: Transverse dorsal arachnoid web and syringomyelia: case report. Neurosurgery 65:E216E217, 2009

11. Tseng SH, Lin SM: Surgical treatment of thoracic arachnoiditis with multiple subarachnoid cysts caused by epidural anesthesia. Clin Neurol Neurosurg 99:256-258, 1997

\section{Author Contributions}

Conception and design: Yamaguchi, Hida. Acquisition of data: Yamaguchi, Takeda, Morishige, Yamada. Analysis and interpretation of data: Yamaguchi, Mitsuhara. Drafting the article: Yamaguchi. Study supervision: Kurisu.

\section{Supplemental Information}

\section{Previous Presentation}

Portions of this work were presented in abstract form at the 5th Annual Meeting of the Cervical Spine Research Society-Asia Pacific Section, Ho Chi Minh City, Viet Nam, April 3-5, 2014.

\section{Correspondence}

Satoshi Yamaguchi, Department of Neurosurgery, Hiroshima University Graduate School of Biomedical and Health Sciences, 1-2-3, Kasumi, Minami-ku, Hiroshima-city, Hiroshima 734-8551, Japan. email: satoya@hiroshima-u.ac.jp. 